\title{
Anatomical popliteal artery entrapment syndrome
}

\author{
Yong Jae Kwon', Tae-Won Kwon', Jun Gyo Gwon², Yong-Pil Cho', Seung-Jun Hwang ${ }^{3}$, Ki-Young Go4 \\ ${ }^{1}$ Division of Vascular Surgery, Department of Surgery, Asan Medical Center, University of UIsan College of Medicine, Seoul, Korea \\ ${ }^{2}$ Department of Surgery, Hanyang University Guri Hospital, Hanyang University College of Medicine, Guri, Korea \\ ${ }^{3}$ Department of Anatomy, Asan Medical Center, University of Ulsan College of Medicine, Seoul, Korea \\ ${ }^{4}$ Department of Radiology, Asan Medical Center, University of Ulsan College of Medicine, Seoul, Korea
}

Purpose: The aim of this study was to analyze anatomical popliteal artery entrapment syndrome (PAES) and to individualize the treatment of this condition according to the anatomical status of the artery and the adjacent structure.

Methods: A total of 35 anatomical PAES legs in 23 consecutive patients treated within the Asan Medical Center, Seoul, Korea between 1995 and 2011 were analyzed retrospectively. Anatomical PAES was diagnosed by MRI and/or CT scans of the knee joint, and CT or conventional transfemoral arteriography of the lower extremities.

Results: We noted a type II gastrocnemius medial head (GNM) anomaly, a type III GNM anomaly, or an aberrant plantaris muscle in $51.4 \%, 20 \%$, and $28.6 \%$ of PAES legs, respectively. In assessments of the arterial lesions, popliteal or tibial artery occlusion was noted in 19 of 26 symptomatic PAES legs. For cases without popliteal artery lesions, myotomy of the anatomically deranged muscle was performed in 5 of 7 symptomatic and 4 of 9 asymptomatic PAES legs. For occluded popliteal arteries, we performed ten direct repairs of the pathological popliteal artery and 4 femoro-below the knee popliteal bypass surgeries. As a result of the arterial Surgery, 9 direct procedures with myotomy yielded a patent artery, while 3 graft failures were noted in the bypass group. The median follow-up period was 84 months (range, 12-206 months). Conclusion: We recommend that treatment of PAES should be individualized based on pathology, symptoms, and various imaging studies.

[Ann Surg Treat Res 2018;94(5):262-269]

Key Words: Plantaris muscle, Popliteal artery

\section{INTRODUCTION}

If promptly diagnosed, a popliteal artery entrapment syndrome (PAES) is the most common surgically correctable cause of leg claudication in young adults [1,2]. A high index of suspicion and the aggressive use of screening tests facilitate the diagnosis of this condition [3,4]. Although the prevalence and natural history of popliteal impingement remains uncertain, it has been proposed that anatomical derangement of the popliteal muscle should be the target of treatment, irrespective of the presence or absence of symptoms because of concerns about the future development of an arterial occlusion [5].

Recent advances in cross-sectional imaging of the knee joint, such as CT and MRI, have yielded a detailed understanding of the anatomy and underlying mechanism of popliteal artery entrapment, allowing the discovery of a new type of PAES associated with an anomaly in the lateral head of the gastrocnemius muscle [6,7]. We have experienced cases of anatomical PAES not related to a gastrocnemius medial head (GNM) anomaly, which have been confirmed to be caused by an aberrant plantaris muscle. Based on this observation, the aim of our present study was to analyze anatomical PAES and to
Received May 30, 2017, Revised August 24, 2017,

Accepted September 12, 2017

Corresponding Author: Tae-Won Kwon

Division of Vascular Surgery, Department of Surgery, Asan Medical Center, University of Ulsan College of Medicine, 88 Olympic-ro 43-gil, Songpa-gu, Seoul 05505, Korea

Tel: +82-2-3010-3492, Fax: +82-2-474-9027

E-mail: twkwon2@amc.seoul.kr

ORCID code: https://orcid.org/0000-0003-3803-0013
Copyright (C) 2018, the Korean Surgical Society

(c) Annals of Surgical Treatment and Research is an Open Access Journal. All articles are distributed under the terms of the Creative Commons Attribution NonCommercial License (http://creativecommons.org/licenses/by-nc/4.0/) which permits unrestricted non-commercial use, distribution, and reproduction in any medium, provided the original work is properly cited. 
individualize the PAES treatments according to the anatomical status of the artery and its adjacent structure.

\section{METHODS}

We retrospectively analyzed 35 PAES legs from 23 consecutive patients who were treated at the Division of Vascular Surgery, Asan Medical Center, Seoul, Korea between 1995 and 2011. This study and the review of patient medical records were approved by the Institutional Review Board of Asan Medical Center (approval number: 2016-0191). MRI or CT scans of the knee joint were used to evaluate musculotendinous anomalies around the popliteal artery. Doppler pressure studies, CT angiography, and/or conventional transfemoral arteriography of the lower extremities were used to evaluate the presence of arterial lesions. In patients with clinically suspected PAES but no demonstrable arterial occlusion upon examination, a provocation test by a forced plantar flexion of the ankle was performed routinely during the Doppler pressure studies, Duplex scans, and conventional angiographic studies.

Patient medical records, Doppler pressure studies, imaging studies of the popliteal artery and adjacent structures (including $\mathrm{CT}, \mathrm{MRI}$ ), and CT angiography or conventional transfemoral arteriography were completely reviewed. We classified the gastrocnemius muscle anomaly, as modified by Rich et al. [3]. Occlusive lesions were classified by their arterial pathology as a segmental occlusion, diffuse occlusion, or distal embolization without popliteal artery occlusion. Nonocclusive lesions were classified as either provocation-positive or -negative based on conventional transfemoral arteriogram findings (Fig. 1). Follow-up with a median duration of 84 months (range, 12-206 months) for all patients was performed at the outpatient clinic and/or by telephone.

\section{RESULTS}

\section{Patients}

A total of 23 patients who experienced muscular derangement of the knee joint and complained of claudication of the leg were identified (Table 1). All patients were physically active with a median age of 28 years old (range, $16-53$ years). There were 26 symptomatic PAES legs in 23 patients with a rate of incidence of symptomatic bilaterality of $13.0 \%$. While studying the contralateral symptomatic legs, we also incidentally identified asymptomatic PAES legs with muscular derangement in nine patients (Table 2). Therefore, the rate of incidence of anatomical bilaterality of PAES was $52.2 \%$ (12 of 23 patients). Regarding the arterial lesions, either popliteal or tibial artery occlusion was noted in 19 of 23 patients (82.6\%) and 19 of 26 symptomatic PAES legs (73.1\%). The symptoms appeared insidiously and repeatedly, but after their symptoms worsened all of the patients visited the hospital within a median duration of 12 months (range, 1-240 months). Although they had visited local clinics or hospitals prior to visiting our hospital, only 4 of 23 patients (17.4\%) visited before occlusion of the popliteal or tibial artery of the affected leg occurred.

\section{Classification}

Among the 26 symptomatic PAES legs, a GNM anomaly was noted in 19 (73.1\%), while an aberrant plantaris muscle was identified in 7 (26.9\%). When we applied the proposal of Rich [3] to classify the lesions, 14 of 19 GNM anomaly legs were classified as type II (73.7\%), and the remaining5 legs (26.3\%) were classified as a type III anomaly. No cases of type I or IV anomalies were identified in our series. If we included asymptomatic legs with anatomical derangements, the type II anomaly, type III anomaly, and aberrant plantaris muscle frequency in our patients were $51.4 \%$ (18 of 35 ), $20 \%$ (7 of 35), and $28.6 \%$ (10 of 35), respectively.
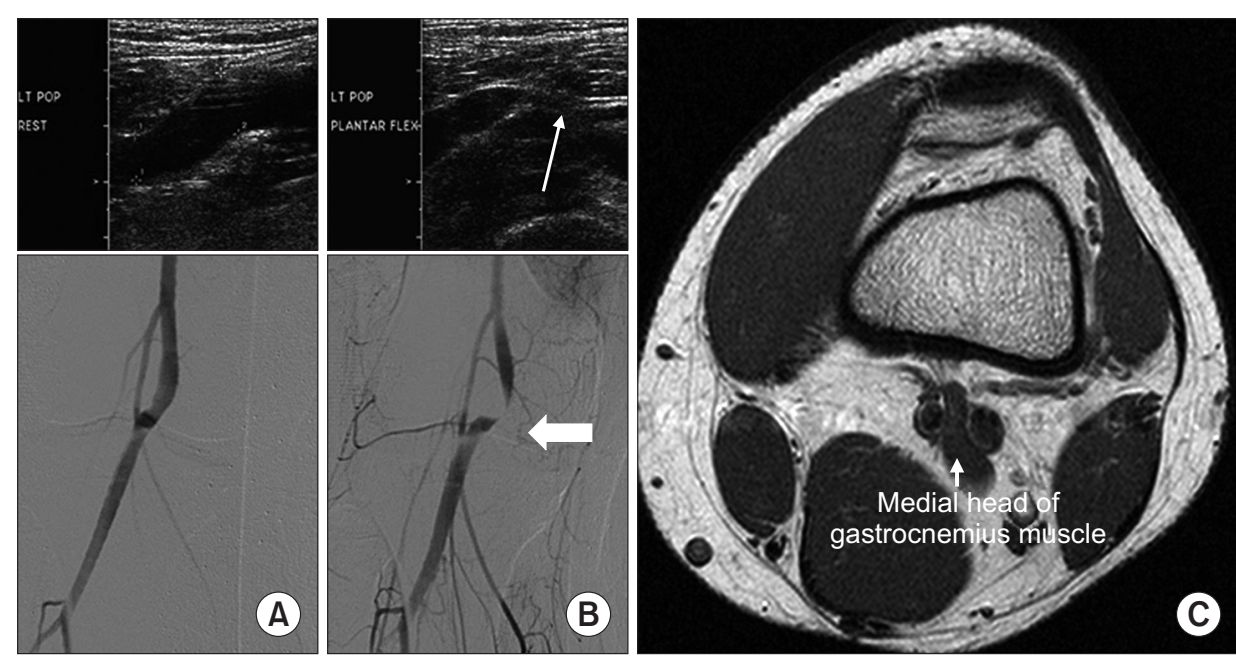

Fig. 1. Ultrasonography $(\mathrm{A}, \mathrm{B}$ : upper panel) and/or conventional transfemoral arteriography (A, B: lower panel) were used in PAES legs with a patent popliteal artery to evoke popliteal artery stenoocclusion using routine plantar flexion of the ankle (the provocation test). The popliteal artery was patent at rest $(A)$, but occlusion of the popliteal artery (white arrow) on plantar flexion of the ankle (B) could be noted in this type II PAES case, which was diagnosed by a MRI of the left knee joint (C) and was determined to be provocationpositive. PAES, popliteal artery entrapment syndrome. 


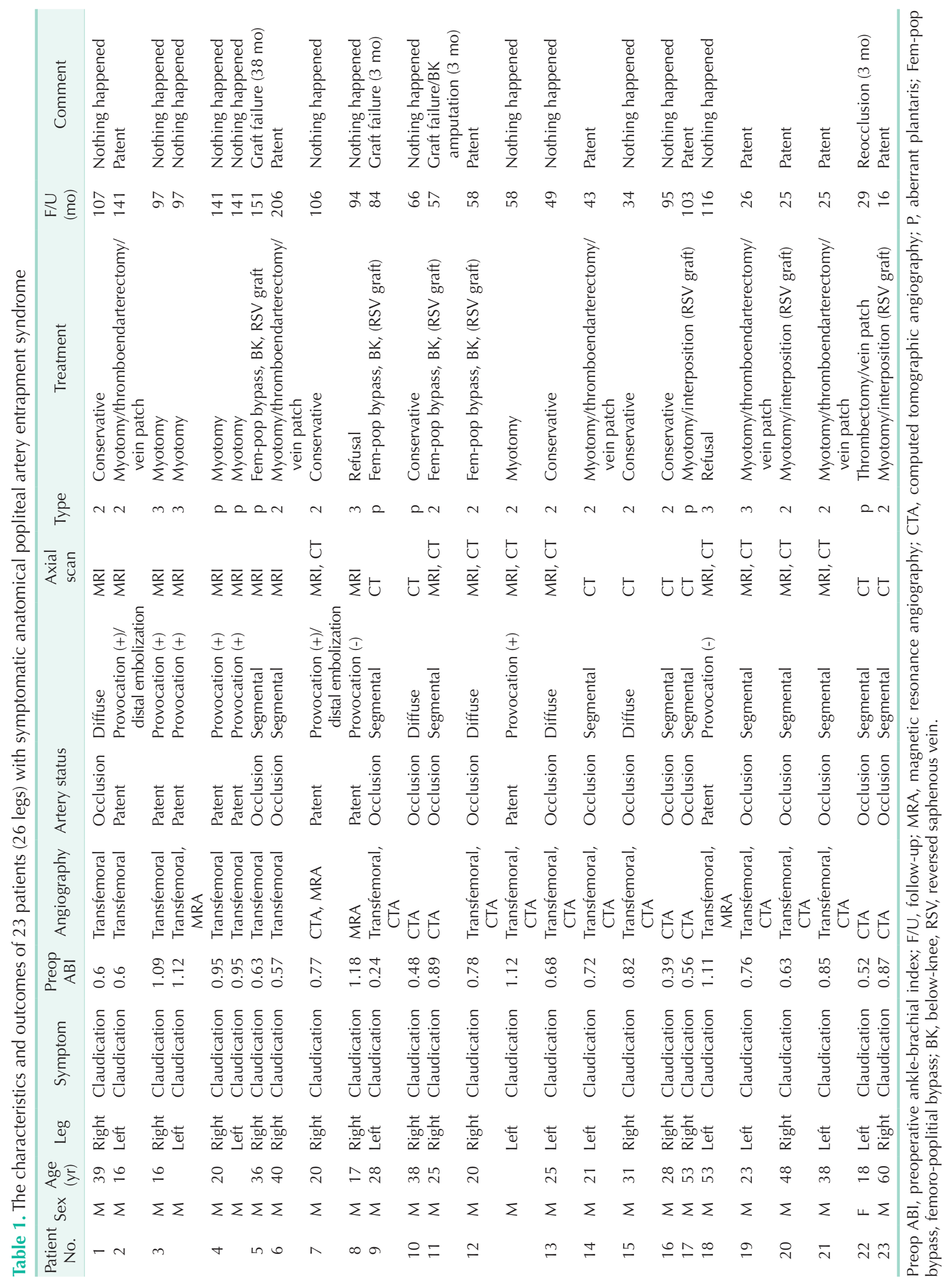


Yong Jae Kwon, et al: Anatomical popliteal artery entrapment syndrome

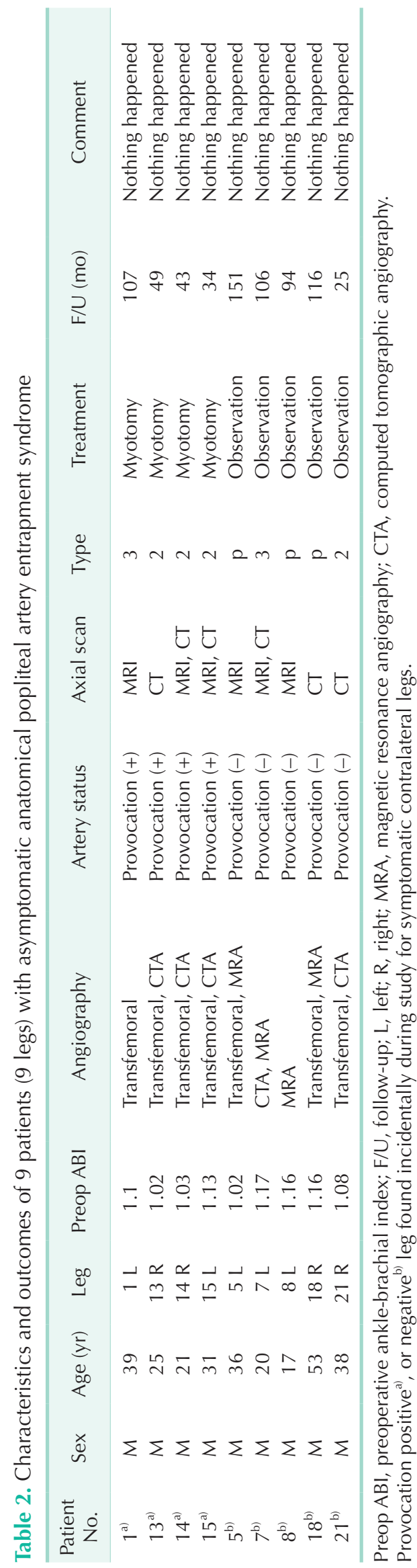

Table 3. Arterial pathology of the popliteal artery according to the type of popliteal artery entrapment syndrome (PAES)

\begin{tabular}{lcccc}
\hline $\begin{array}{c}\text { Type of } \\
\text { PAES }\end{array}$ & $\begin{array}{c}\text { Aberrant plantaris } \\
\text { muscle }(\mathrm{n}=7)\end{array}$ & $\begin{array}{c}\text { Type II } \\
(\mathrm{n}=14)\end{array}$ & $\begin{array}{c}\text { Type III } \\
(\mathrm{n}=5)\end{array}$ & P-value \\
\hline Occlusion & $5(71.4)$ & $13(92.9)$ & $1(20.0)$ & 0.007 \\
Patent & $2(28.6)$ & $1(7.1)$ & $4(80.0)$ & \\
\hline
\end{tabular}

Values are presented as number (\%).

Regarding arterial pathology and the mechanism of popliteal artery entrapment, an occlusion was noted in 13 of 14 type II GNM anomalies (92.9\%), 1 of 5 type III GNM anomalies (20\%), and 5 of 7 aberrant plantaris muscle cases (71.4\%). Therefore, a type II anomaly appeared to be significantly more complex in its course than any other type $(P=0.007)$ (Table 3). We observed no differences in findings for patients who underwent CT or MR arteriography versus transfemoral arteriography of the lower extremities (Fig. 2).

\section{Description of PAES caused by an aberrant plantaris muscle}

All aberrant plantaris muscles were higher and more medially located than a normal plantaris muscle, and these abnormal muscles ran along or across the popliteal artery in the upper portion of the popliteal fossa in both CT and MRI scans of the affected knee joints. This location can cause occlusion of the popliteal artery during forced plantar flexion of the ankle, and an occlusion was demonstrated by a provocation study during a transfemoral arteriography of the lower extremity that revealed a patent popliteal artery at rest but occlusion of the popliteal artery during plantar flexion of the foot. We confirmed this finding by passive plantar flexion of the foot during the operation (Fig. 3).

\section{Arterial pathology and provocation test}

Assessment of the pattern of occlusion revealed that segmental occlusion of the popliteal artery was the most common with an incidence of $63.2 \%$ (12 of 19). The rates of incidence of diffuse occlusion and distal embolization were $26.3 \%$ (5 of 19) and $10.5 \%$ (2 of 19), respectively. The popliteal artery was explored during an operation in 14 of 26 affected legs, including 9 occluded and 5 patent arteries. For the 9 occluded lesions, a chronic occlusion with an organized thrombus was noted in 5 lesions and was the most common pattern. Additionally, operative findings revealed acute thrombosis, stent failure with thrombosis, luminal ulceration with distal embolization, and a thrombosed popliteal artery aneurysm in one leg each. Using a provocation test involving conventional transfemoral arteriography to evoke symptoms for legs with a patent popliteal artery, 5 provocation-positive legs $(71.4 \%)$ and 2 provocation-negative legs $(28.6 \%)$ were noted among the 7 

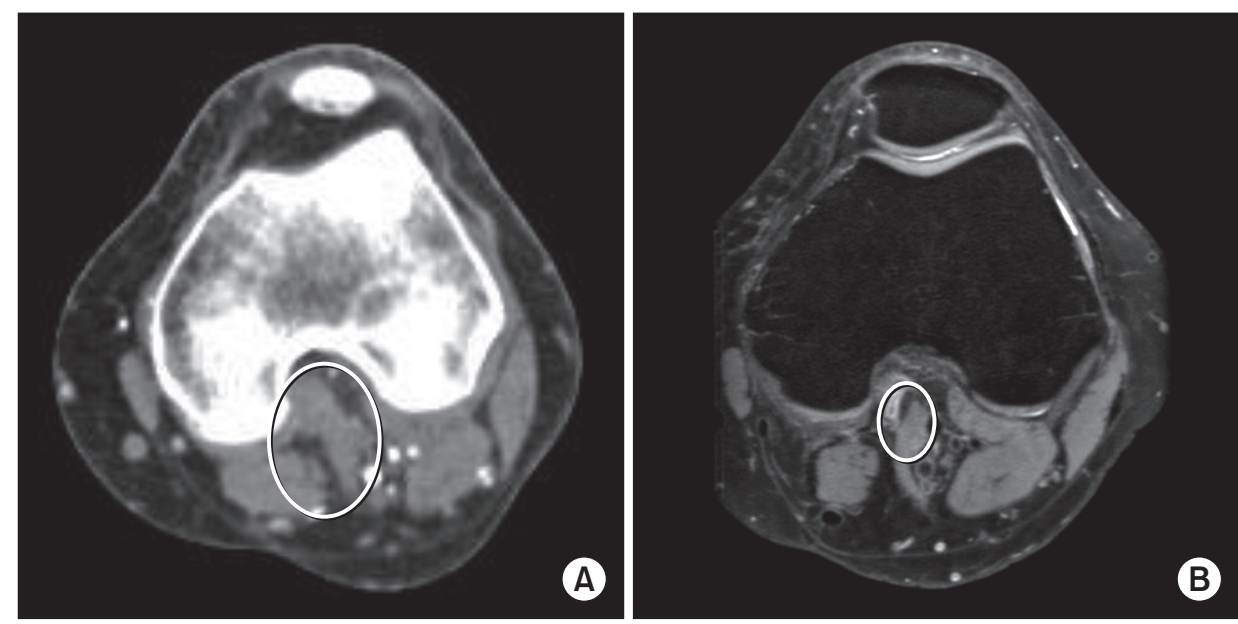

Fig. 2. MRI and CT scan of the knee joint. There was no discrepancy in the findings from the $\mathrm{CT}$ scan (A) and the MRI (B). Medial ahead of gastrocnemius muscle with arterial occlusion could be observed in the left leg (A, B: circle).
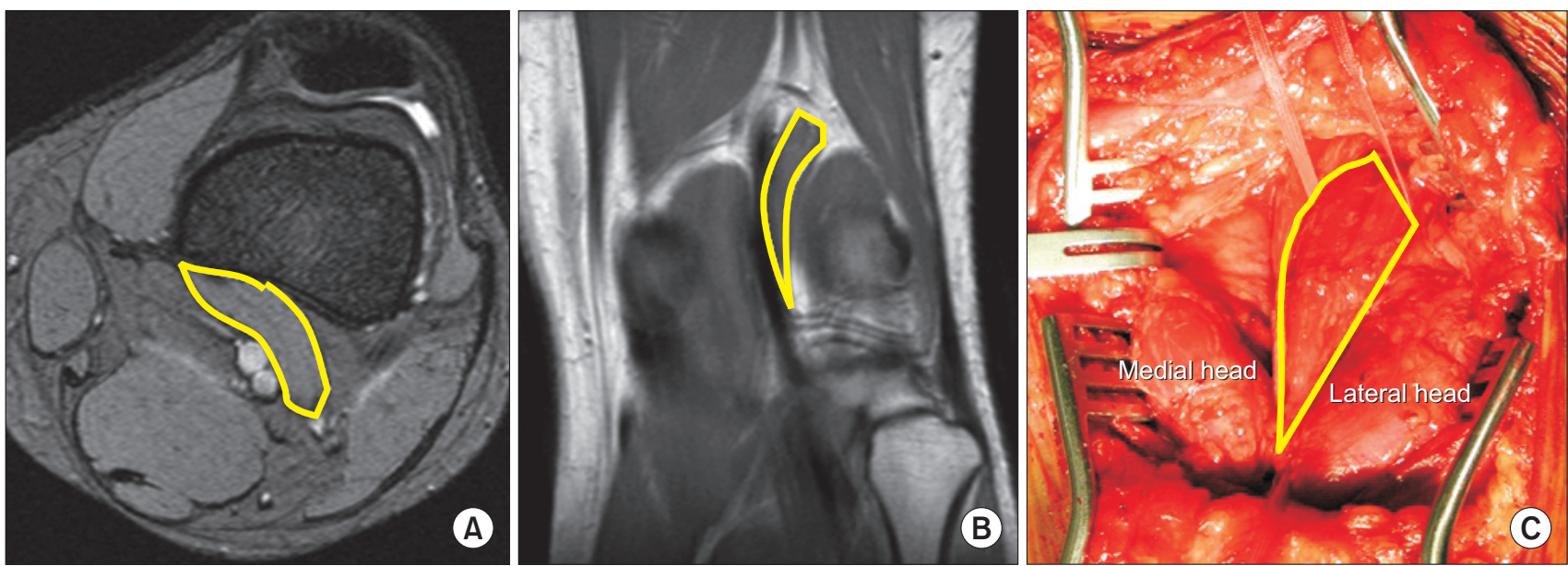

Fig. 3. T1-weighted axial (A) and coronal section (B) of the knee joint showed aberrant plantaris muscle which originated more medially and higher than normal position, that we confirmed on operation (C).

symptomatic legs. In contrast, among nine asymptomatic legs, 4 legs (44.4\%) were provocation-positive, and 5 legs (55.6\%) were provocation-negative. When we analyzed the associations between symptoms and provocation positivity or negativity, 4 of 9 provocation-positive legs (44.4\%) legs with muscular derangement were asymptomatic, while 2 of 7 provocationnegative legs (28.6\%) with muscular derangement were symptomatic. These findings indicated that not all cases that were positive by the provocation test showed symptoms, and, surprisingly, cases that were negative by the provocation test can still exhibit symptoms.

\section{Treatments}

Treatments were selected according to the arterial pathology and symptoms. Within the seven symptomatic PAES legs with a patent popliteal artery, myotomy of the anatomically deranged muscle was performed in all cases that were provocationpositive, and the 2 individuals who refused treatment were provocation-negative. We routinely used a posterior approach for the myotomy. The symptoms of intermittent claudication disappeared in all of the patients with PAES legs with a patent popliteal artery who underwent myotomy of the anatomically deranged muscle. The typical symptoms and no arterial pathologies appeared during the study period in any of the patients who refused myotomy. Among the nine asymptomatic but incidentally identified PAES legs, we performed myotomy only when the provocation study showed a positive result (4 legs). Moreover, no leg symptoms or arterial pathologies appeared during the study period in any patient in this asymptomatic group.

For occluded popliteal arteries, arterial surgery was performed in 14 of 19 PAES legs, while conservative treatment was conducted in 5 legs. We performed direct repair of the pathological popliteal artery via a posterior approach in 10 legs, which consisted of 6 cases of thromboendarterectomy with saphenous vein patch angioplasty, 3 cases of graft interposition 
Yong Jae Kwon, et al: Anatomical popliteal artery entrapment syndrome

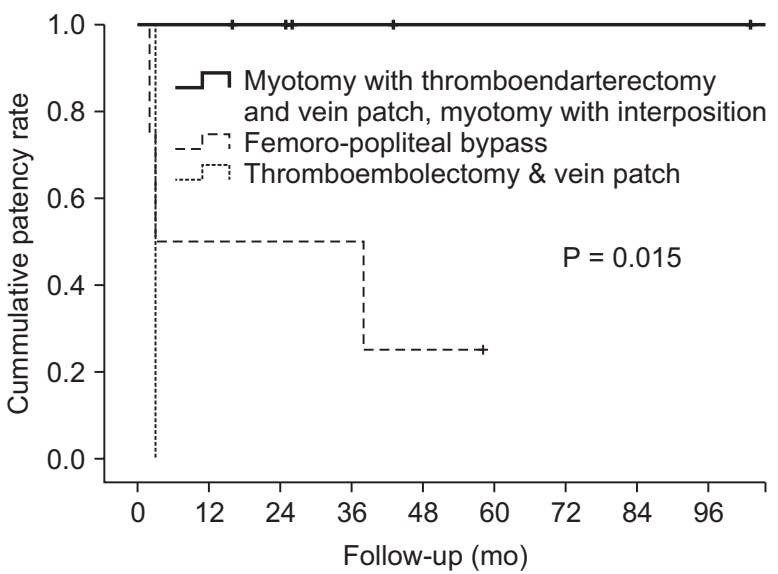

Fig. 4. Patency rates according to the treatment methods for symptomatic popliteal artery entrapment syndrome.

using a reverse saphenous vein graft followed by myotomy of the anatomically deranged muscle, and 1 case of thrombectomy with a saphenous vein patch angioplasty. Femoro-below the knee popliteal bypass surgery using a reverse saphenous vein graft, which was subcutaneously placed, was performed in four PAES legs. All 9 direct procedures with myotomy yielded a patent artery during the study period, while 1 direct procedure without myotomy resulted in a thrombotic reocclusion of the popliteal artery in a pattern similar to the original lesion. Bypass surgery was associated with 3 graft failures, including 2 early graft failures and 1 limb loss (Fig. 4).

\section{DISCUSSION}

The actual rate of incidence of anatomical PAES is unknown but it appears to be more common than previously appreciated [8]. There is a growing awareness that PAES can be a cause of claudication, and the use of noninvasive diagnostic techniques, including stress tests, have contributed to its increasing incidence [9]. In our present study series, patients had visited local clinics or hospitals prior to visiting our hospital, but only 4 of 23 patients (17.4\%) and 7 of 26 legs (26.9\%) presented prior to the onset of occlusion of the anatomically entrapped popliteal artery. Thus, this series emphasizes the importance of a high index of suspicion and aggressive screening tests for the early diagnosis of PAES.

A provocation test can aid the diagnosis of patients with less apparent manifestations. In suspected cases of PAES, the provocation test can help differential diagnosing of PAES. According to the current understanding of PAES pathogenesis, recurrent compression of the popliteal artery causes physical damage to the vessel wall, which can lead to luminal narrowing, the formation of aneurysms, thrombosis, or distal embolization [10]. Through performing surgery, we have encountered all of the arterial pathologies that can result from entrapment of the popliteal artery by an anatomically deranged muscle during passive plantar flexion of the ankle.

We also found that we could classify the arterial lesions in our current study series based on their imaging characteristics. Occlusive lesions were classified as a segmental occlusion, segmental occlusion with distal extension (diffuse), or a patent popliteal artery with distal embolization. Additionally, nonocclusive lesions were divided into provocation-positive or -negative categories by a transfemoral arteriogram.

Anatomical PAES has been previously classified into various types based on the abnormal relationship between the GNM and popliteal artery in the popliteal fossa [3]. Advances in understanding of the developmental embryology and mechanism of impingement of the popliteal artery have resulted in modifications to this classification [11]. However, MRI and CT scans of the knee joint have recently provided more precise anatomical details of the popliteal region, and new types of PAES have been introduced as a consequence [12]. Anomalies of the lateral head of the gastrocnemius muscle can play a role in the development of PAES, and Kim [7] classified such anomalies into 2 types-an abnormal insertion type and an aberrant accessory slip type. We experienced some cases of PAES that were related to an aberrant plantaris muscle rather than a gastrocnemius muscle anomaly. At the time when PAES was introduced, many authors had reported encountering the plantaris muscle in PAES in their operative findings [13]. Indeed, the plantaris muscle is a rudimentary structure in humans and can be considered to be an accessory to the lateral head of the gastrocnemius muscle. However, when the plantaris muscle develops abnormally, it can entrap the popliteal artery upon contraction, which occurs upon plantar flexion of the ankle [14]. In our present case series, $26.9 \%$ of anatomical PAES limbs were associated with an aberrant plantaris muscle, a more frequent occurrence than we expected before initiating this study. All aberrant plantaris muscles originated more medially and superior than the normal position, ran obliquely, and crossed the popliteal artery at the popliteal fossa on both MRI and CT scans. Therefore, the importance of the aberrant plantaris muscle as a cause of PAES should be emphasized. Although we could not extend the classification of GNM anomalies beyond the traditionally used criteria, we believe that an alternative classification for PAES could be derived that is based on careful examination of imaging findings, such as MRI or CT scans.

Type II anomalies showed a higher incidence of arterial occlusion compared to type III anomalies. We suspect that when the artery abnormally inserts into the medial head of the gastrocnemius muscle, this position results in stronger compressive forces on the popliteal artery compared with the accessory slip or aberrant head. Additionally, in our analysis of the relationship between provocation tests and asymptomatic incidentally detected anatomical derangement of the knee 


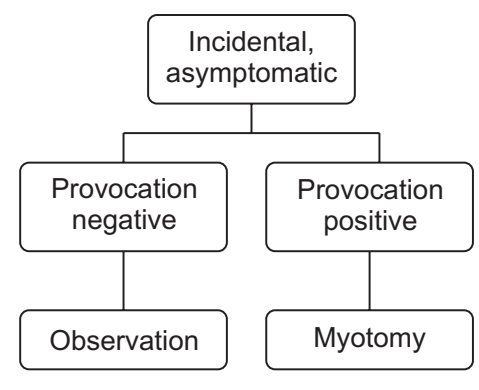

Incidental,

symptomatic

Provocation

Observation

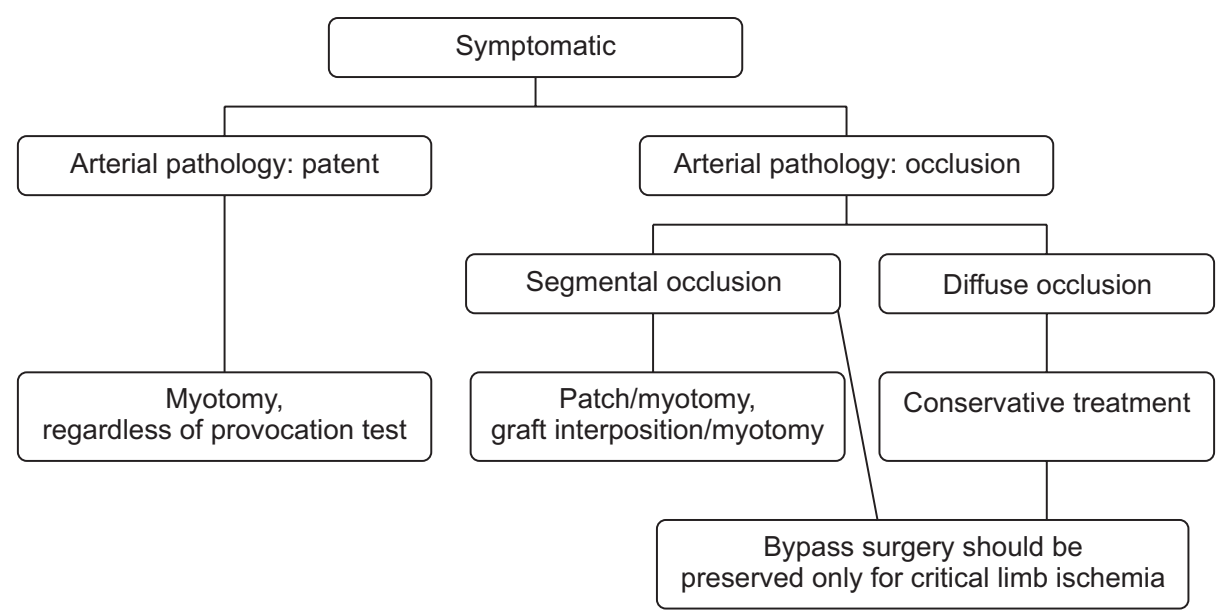

Fig. 5. Suggestions for the individualized treatment of PAES. Treatment based on provocation tests for patients with asymptomatic PAES legs is recommended, and symptomatic PAES legs should be treated based on the arterial pathology. PAES, popliteal artery entrapment syndrome.

joint muscle the provocation test yielded negative results in all 3 aberrant plantaris muscle group cases compared to $50 \%$ positivity in the GNM anomaly group. Specifically, our present series included 9 PAES legs that tested as provocationpositive on the transfemoral arteriogram among 16 legs with an anatomically deranged muscle around the knee joint in the absence of a popliteal artery occlusion. Murray et al. [13] demonstrated that not all cases of anatomical PAES exhibit symptoms. This observation is further supported by the incidental detection of anatomically impinged popliteal arteries in the asymptomatic contralateral limb, which is not an uncommon finding. Therefore, the actual prevalence and natural history of popliteal impingement remains uncertain and should be elucidated in future studies [15].

Surgically, we routinely used a posterior approach for myotomy as recommended by many authors. To identify the muscle anomaly and any abnormal relationship between the popliteal artery and gastrocnemius or other muscles, including the plantaris muscle,was important.

Our general principle for the treatment of occlusive arterial lesions is that below the knee bypass surgery should only be performed in patients with critical limb ischemia. For PAES, however, we performed operative interventions for segmental occlusion of the popliteal artery while we conducted conservative treatment in most cases of diffuse arterial occlusion without critical limb ischemia. The result of this direct approach was better than bypass surgery. We propose that direct repair of a popliteal artery lesion followed by myotomy via a posterior approach could have the best chance of achieving graft patency. Furthermore, 1 case in our series, in whom we conducted only thrombectomy without myotomy of the plantaris muscle via a posterior approach, resulted ultimately in reocclusion of the popliteal artery in a pattern identical to the original lesion at 3 months after the operation. Thus, when direct repair of the popliteal artery via a posterior approach is performed, myotomy of the anatomically deranged muscle should follow.

The optimal treatment of incidentally detected asymptomatic popliteal artery entrapment with deranged muscle anatomy is controversial. Most authors argue that an anatomically deranged muscle should be treated irrespective of the symptoms because an arterial occlusion will develop [15]. However, our policy is to perform myotomy of the abnormally inserted gastrocnemius muscle or aberrant plantaris muscle only in cases of provocation-positive legs and to only observe cases of provocation-negative legs in asymptomatic patients. We have established that our approach is safe because there was no relapse during the follow-up period with a median of 106 months for the 5 provocation-negative legs we treated.

In conclusion, treatment of PAES should be individualized based on pathology, symptoms and imaging studies. Imaging studies using a CT scan or MRI with various reconstruction methods may indicate muscle abnormalities to plan and perform proper treatment. Based on our provocation study, treating asymptomatic legs with an anatomically deranged muscle may be advisable (Fig. 5). When the occlusive lesion is confined to the popliteal artery, direct repair of the popliteal artery followed by myotomy of the anatomically deranged muscle via a posterior approach is recommended.

\section{CONFLICTS OF INTEREST}

No potential conflict of interest relevant to this article was reported. 


\section{REFERENCES}

1. Insua JA, Young JR, Humphries AW. Popliteal artery entrapment syndrome. Arch Surg 1970;101:771-5.

2. Delaney TA, Gonzalez LL. Occlusion of popliteal artery due to muscular entrapment. Surgery 1971;69:97-101.

3. Rich NM, Collins GJ Jr, McDonald PT, Kozloff L, Clagett GP, Collins JT. Popliteal vascular entrapment. Its increasing interest. Arch Surg 1979;114:1377-84.

4. di Marzo L, Cavallaro A, Sciacca V, Mingoli A, Tamburelli A. Surgical treatment of popliteal artery entrapment syndrome: a ten-year experience. Eur J Vasc Surg 1991; 5:59-64.

5. Turnipseed WD. Popliteal entrapment syndrome. J Vasc Surg 2002;35:910-5.

6. Liu PT, Moyer AC, Huettl EA, Fowl RJ, Stone WM. Popliteal vascular entrapment syndrome caused by a rare anomalous slip of the lateral head of the gastrocnemius muscle. Skeletal Radiol 2005;34:359-63.

7. Kim HK, Shin MJ, Kim SM, Lee SH, Hong HJ. Popliteal artery entrapment syndrome: morphological classification utilizing MR imaging. Skeletal Radiol 2006;35:648-58.

8. Levien LJ, Veller MG. Popliteal artery entrapment syndrome: more common than previously recognized. J Vasc Surg 1999:30:587-98.

9. Pillai J, Levien LJ, Haagensen M, Candy G, Cluver MD, Veller MG. Assessment of the medial head of the gastrocnemius muscle in functional compression of the popliteal artery. J Vasc Surg 2008:48:1189-96.

10. Turnipseed WD, Pozniak M. Popliteal entrapment as a result of neurovascular compression by the soleus and plantaris muscles. J Vasc Surg 1992;15:285-93.

11. di Marzo L, Cavallaro A. Popliteal vascular entrapment. World J Surg 2005;29 Suppl 1: S43-5.

12. Kwon YJ, Kwon TW, Um EH, Shin S, Cho YP, Kim JM, et al. Anatomical popliteal artery entrapment syndrome caused by an aberrant plantaris muscle. Vasc Specialist Int 2015:31:95-101.

13. Murray A, Halliday M, Croft RJ. Popliteal artery entrapment syndrome. Br J Surg 1991;78:1414-9.

14. Chernoff DM, Walker AT, Khorasani R, Polak JF, Jolesz FA. Asymptomatic functional popliteal artery entrapment: demonstration at MR imaging. Radiology 1995;195:176-80.

15. Collins PS, McDonald PT, Lim RC. Popliteal artery entrapment: an evolving syndrome. J Vasc Surg 1989;10:484-9. 